\title{
Folic acid-conjugated mesoporous silica nanoparticles for enhanced therapeutic efficacy of topotecan in retina cancers
}

This article was published in the following Dove Press journal: International Journal of Nanomedicine

\author{
Wei Qu' \\ Bo Meng' \\ Yangyang $\mathrm{Yu}^{2}$ \\ Shaowei Wang' \\ 'Department of Ophthalmology, \\ The 2nd Affiliated Hospital of Harbin \\ Medical University, ${ }^{2}$ Department \\ of Ophthalmology, Heilongjiang \\ Provincial Hospital, Harbin, \\ Heilongjiang, People's Republic \\ of China
}

\begin{abstract}
In this study, topotecan-loaded mesoporous silica nanoparticles were prepared and surface conjugated with folic acid (FTMN) to enhance the therapeutic efficacy of topotecan for the treatment of retinoblastoma (RB) cancers. The particles were nano-sized and exhibited a sustained release of drug in the physiological conditions. The folic acid-conjugated nanoformulations exhibited a remarkable uptake in RB cells compared to that of non-targeted nanoparticles. These results clearly indicate that receptor-mediated endocytosis is the mechanism of cellular internalization. The greater cellular uptake of FTMN resulted in significantly higher cytotoxic effect in Y79 cancer cells compared to that of other formulations. The results were well corroborated with the live/dead assay and nuclear fragmentation assay. FTMN consistently induced apoptosis of cancer cells with an efficiency of $\sim 58 \%$. Our results clearly showed that nanoparticulate encapsulation of TPT exhibited superior anticancer efficacy in Y79 cancer cells compared to that of free drug or non-targeted nanoparticles. As expected, FTMN exhibited a remarkable reduction in the overall tumor volume compared to any other group with less presence of tumor cells in histology staining. Overall, folic acid-conjugated nanoparticulate system could provide an effective platform for RB treatment.
\end{abstract}

Keywords: retinoblastoma, topotecan, mesoporous silica nanoparticles, apoptosis, anticancer effect

\section{Introduction}

Retinoblastoma (RB) is a rare tumor of the eye which occurs in infants and children. The incidence of RB is rare it affects only about 1 in 20,000 children. ${ }^{1}$ The onset of $\mathrm{RB}$ results in either vision loss or secondary tumor development. It is associated with the mutation of $\mathrm{RB}$ gene $(R B 1)$ in about $40 \%$ of patients with $\mathrm{RB}^{2,3}$ The cure rate of $\mathrm{RB}$ is more than $90 \%$ in developed countries; however, secondary tumor development after many years of treatment is a severe problem. Current treatment regimens for RB include local thermotherapy, cryotherapy, and radiotherapy. ${ }^{4}$ However, it has been reported that such therapies result in severe complications including cataract, retinopathy, and facial deformities. To effectively reduce the tumor burden, primary chemotherapy is often necessary along with other local treatments. Although systemic administration of chemotherapeutic drugs reduced the incidence of $\mathrm{RB}$, it resulted in severe systemic toxicity to normal organs, necessitating the need to revise the treatment modality. ${ }^{5,6}$

In this context, effective delivery of anticancer drugs to the desired tumor region without exhibiting its side effects would be an interesting prospect. In cases of RB, the ideal situation would be to deliver the drugs to the eye without causing any side
Correspondence: Shaowei Wang Department of Ophthalmology, The 2nd Affiliated Hospital of Harbin Medical University, Harbin, Heilongjiang 15008,

People's Republic of China

Tel/fax +86 45I 8666296 I

Email shaoweiwang191@hotmail.com 
effects to other parts of the eye as well as to the systemic regions. ${ }^{7}$ Topotecan (TPT) is a topoisomerase 1 inhibitor which breaks the DNA strands and inhibit the cell replications with proven therapeutic activity against many cancers including RB. ${ }^{8,9}$ It has been reported that the controlled release of TPT to the tumor tissues would be more beneficial and that high concentrations of drug should be delivered to the tumor without causing any adverse effects. ${ }^{10,11}$ Therefore, delivering increased concentration of TPT to the eye via nanoparticles (NPs) might be an interesting strategy for further research.

All of the limitations of a traditional chemotherapeutic drug may be effectively overcome by the formulation of NPs. ${ }^{12}$ Drug encapsulation in NPs might help to overcome the problem associated with the blood-retina barrier. Among the plethora of NP systems, mesoporous silica nanoparticles (MSNs) offer several attractive features such as large surface area, nano-sized particles, surface modifications, pore size and pore shape, large-scale synthesis, and excellent colloidal stability. ${ }^{13-15}$ The drug could be easily loaded in the pores of the NPs and thereby prevent from the premature release and maintain the integrity of the encapsulated drugs. ${ }^{16,17}$ It has been reported that surface modification of NPs with suitable ligands or targeting agents might improve the therapeutic efficacy and reduce the unwanted side effects. ${ }^{18}$ Conjugation of NPs with antibodies and ligands such as folic acid (FA) allows for the specific targeting of NPs to the cancer cells which overexpresses the receptor for the targeting ligand. ${ }^{19}$ In this regard, studies have repeatedly shown that FA is an attractive targeting agent which is very specific to the FA receptors $\left(\mathrm{K}_{\mathrm{d}} \sim 10^{-10} \mathrm{M}\right)$. FA receptors are overexpressed in several cancers such as in breast, brain, and lung cancers and in RB. It is worth noting that the expression level of FA receptors is around 150-300 times more than that of the normal tissues. ${ }^{20,21}$ Owing to this distinguishing property, FA has emerged as a suitable targeting agent for the cancer treatment.

In this study, we aimed to increase the anticancer efficacy of TPT in the treatment of RB. For this purpose, TPT-loaded MSNs (TMNs) were prepared and surface conjugated with FA (FTMNs). The TMNs and FTMNs were characterized for particle size and size distributions and release kinetics. Targeting specificity of FA toward RB cells was studied by means of cellular uptake analysis (fluorescence-activated cell sorter [FACS] and confocal laser-scanning microscopy [CLSM]). The anticancer effect of individual formulations was evaluated by means of cytotoxicity assay, live/dead assay, Hoechst 33342 staining, and apoptosis assay.

\section{Materials and methods Materials}

Cetyltrimethylammonium bromide, tetraethylorthosilicate, aminopropyl-triethoxysilane (APTES), FA, and TPT were purchased from Sigma-Aldrich Co. (St Louis, MO, USA). All other chemicals were of reagent grade and used as such.

\section{Preparation of FA-conjugated MSNs}

First, to prepare MSN, $500 \mathrm{mg}$ of cetyltrimethylammonium bromide (CTAB) was dissolved in $240 \mathrm{~mL}$ water and stirred well. To this CTAB solution, $1.8 \mathrm{~mL}$ of $2 \mathrm{M} \mathrm{NaOH}$ was added and the temperature was increased to $80^{\circ} \mathrm{C} .5 \mathrm{~mL}$ of ethanolic tetraethylorthosilicate $(21.9 \mathrm{mmol})$ was added to the above solution and stirred for $3 \mathrm{~h}$. After the solution was cooled, particles were separated by filtration and dried. The particles were repeatedly washed with hydrochloric acid and methanol to remove the surfactant residues and refluxed for $24 \mathrm{~h}$. The particles were finally washed with water and dried and kept aside. Separately, $450 \mathrm{mg}$ of FA, $200 \mathrm{mg}$ of $\mathrm{N}, \mathrm{N}^{\prime}$-dicyclohexylcarbodiimide, and $180 \mu \mathrm{L}$ of APTES was dissolved in $35 \mathrm{~mL}$ of anhydrous DMSO and stirred for $24 \mathrm{~h}$ under inert atmosphere. The reactive FA was reacted with $1 \mathrm{~g}$ of MSN and refluxed for $4 \mathrm{~h}$ at $110^{\circ} \mathrm{C}$. The final product was washed and dried overnight.

\section{Particle size and morphology}

The average particle size, polydispersity index, and surface charge were evaluated using Zetasizer instrument (NanoZ590, Malvern Instruments Ltd, Malvern, Worcestershire, UK). The suspension was diluted with distilled water and studied at $25^{\circ} \mathrm{C}$. The experiment was performed in triplicate. The morphology of particles was evaluated using transmission electron microscopy (JEM 2100F, JEOL, Tokyo, Japan). The suspension was stained with $2 \%$ phosphotungistic acid and placed on a copper grid. The sample was dried and observed under microscope.

\section{In vitro drug release}

The in vitro drug release study was performed by dialysis protocol. A dialysis bag with an average molecular weight of 5,000 Da was used to load the drug-loaded formulations. The dialysis bag was sealed and put in a centrifuge tube containing $25 \mathrm{~mL}$ of the release media. The set-up was placed in a shaker bath maintained at $37^{\circ} \mathrm{C}$ and $100 \mathrm{rpm}$. At specific time, $2 \mathrm{~mL}$ of the media was taken out and fresh media was added to maintain the sink conditions. The amount of drug released in the media was calculated by HPLC method. 


\section{Cell culture conditions}

RB cells (Y79; ATCC; Manassas, VA, USA) were grown in RPMI culture medium supplemented with $10 \%$ FBS and $1 \%$ antibiotic mixture. The cells were grown in ambient conditions under humidified atmosphere.

\section{In vitro cellular uptake of NPs}

To investigate the cellular uptake pattern of targeted and non-targeted NPs, rhodamine-B was loaded in the MSNs and purified by gel filtration chromatography using Sephadex G-50 column. Y79 cells were seeded in a 6-well plate at a seeding density of $2 \times 10^{5}$ cells/well and the cells were kept aside for $24 \mathrm{~h}$. The cells were then treated with TMN and FTMN and incubated for $2 \mathrm{~h}$ in the RPMI media. Followed by this, cells were washed three times with PBS and fixed by paraformaldehyde. The cells were stained with $4^{\prime}$, 6-diamidino-2-phenylindole to stain the nucleus. Then, the cells were washed again and observed through CLSM (Nikon, Tokyo, Japan).

The cellular uptake efficiency was further studied by flow cytometer (FACS; BD Biosciences, San Jose, CA, USA). Y79 cells were seeded in a 6-well plate at a density of $2 \times 10^{5}$ cells/ well and the cells were kept aside for $24 \mathrm{~h}$. The cells were then treated with TMN and FTMN and incubated for $2 \mathrm{~h}$ in the RPMI media. The cells were extracted and washed two times with PBS. Then, the cells were centrifuged and the pellet was re-dispersed in PBS and detected by flow cytometer.

\section{Cytotoxicity assay}

The cytotoxic effect of free TPT, TMN, and FTMN was evaluated by 3-(4, 5-dimethylthiazol-2-yl)-2, 5-diphenyl tetrazolium bromide (MTT) assay. The MTT assay works on the basis of cleavage of tetrazolium salt into insoluble formazan crystals, whose absorbance is measured. The Y79 cells were seeded in 96-well plate at a seeding density of $1 \times 10^{4}$ cells/well and the cells were kept aside for $24 \mathrm{~h}$. The cells were then treated with free TPT, TMN, and FTMN and kept aside for $24 \mathrm{~h}$. Separately, blank NPs were incubated in cells at increasing concentration. The medium was removed the next day and treated with MTT reagent and incubated for $4 \mathrm{~h}$. The media was carefully removed and treated with $100 \mu \mathrm{L}$ of DMSO to extract formazan crystal. Absorbance of formazan in individual plate was read using microplate reader (BioTek Instruments, Winooski, VT, USA) at $570 \mathrm{~nm}$. Statistical analysis was performed using one-way analysis of variance and Student's $t$-test with $p<0.05$ is considered statistically significant.

\section{Nuclear morphology}

The Y79 cells were seeded in a 6-well plate containing cover slips at a seeding density of $2 \times 10^{5}$ cells/well and the cells were kept aside for $24 \mathrm{~h}$. Then, the cells were treated with free TPT, TMN, and FTMN and kept aside for $24 \mathrm{~h}$. Next day, cells were fixed with paraformaldehyde for $10 \mathrm{~min}$. The cells were then washed two times and stained with Hoechst $3342(10 \mu \mathrm{g} / \mathrm{mL})$ for $15 \mathrm{~min}$. The cells were again washed with PBS, and the cover slips were mounted on the glass slides. The nuclear morphology of cancer cells were observed using fluorescence microscope.

\section{Live/dead assay}

The live/dead assay was performed by acridine orangeethidium bromide double staining protocol. The cells were then treated with free TPT, TMN, and FTMN and kept aside for $24 \mathrm{~h}$. Then, the cells were harvested and washed properly with PBS. Then, the harvested cells were stained with acridine orange-ethidium bromide staining kit as per the manufacturer's protocol and observed under fluorescence microscope.

\section{Annexin V/propidium iodide-based apoptosis assay}

The Y79 cells were seeded in a 12-well plate containing cover slips at a density of $2 \times 10^{5}$ cells/well and the cells were kept aside for $24 \mathrm{~h}$. Then, the cells were treated with free TPT, TMN, and FTMN and kept aside for $24 \mathrm{~h}$. The cells were washed and harvested by scraping. The harvested cells were washed and pelleted. Next, the cell pellets were resuspended in a $100 \mu \mathrm{L}$ of binding buffer and stained with $2.5 \mu \mathrm{L}$ of annexin $\mathrm{V}$ and $2.5 \mu \mathrm{L}$ of propidium iodide and incubated for $15 \mathrm{~min}$. The volume was made up to $1 \mathrm{~mL}$ and the samples were analyzed using flow cytometer at a rate of 10,000 events.

\section{Antitumor efficacy study}

The animal experimental protocol was approved by the Institutional Animal Ethical Member Board of The 2nd Affiliated Hospital of Harbin Medical University, People's Republic of China. We have followed the guidelines for Care and Use of Laboratory Animals, China. The tumor was developed in nude mice (Y79 cells) and then equally divided into 4 groups. The mice were injected with respective formulation and tumor volume was evaluated by vernier caliper. The tumor was extracted and further subjected to histological analysis. 


\section{Statistical analysis}

The statistical analysis was performed by Student's $t$-test and a $p<0.05$ was considered statistically significant. All the data are presented as mean \pm standard deviation.

\section{Results and discussion}

$\mathrm{RB}$ is a rare tumor of the eye which occurs in infants and children. In this study we synthesized MSNs and surface conjugated with FA to enhance the anticancer efficacy of TPT in RB. The conjugation with antibodies and ligands such as FA allows for the specific targeting of NPs to the cancer cells which overexpresses the receptor for the targeting ligand (Figure 1). The conjugation of FA with the MSN was evaluated by FTIR (Figure S1). First, FTIR spectra of blank MSN was observed followed by the FTIR spectra of MSN-NH after the addition of APTES in the reaction mixture. The peaks around $1,650 \mathrm{~cm}^{-1}$ and $1,460 \mathrm{~cm}^{-1}$ indicate the presence of $\mathrm{N}-\mathrm{H}$ and $\mathrm{C}-\mathrm{N}$ stretching vibrations suggesting that APTES had introduced the amine functional group on the surface of MSN. In addition, $\mathrm{CH}_{2}$ stretching vibrations were observed around $3,000 \mathrm{~cm}^{-1}$. FTIR spectra clearly indicate the successful conjugation of FA to the MSN. As seen, $\mathrm{NH}_{2}$ peaks around 1,650 $\mathrm{cm}^{-1}$ was disappeared after FA conjugation while new peaks were observed at $1,660 \mathrm{~cm}^{-1}$ and $1,600 \mathrm{~cm}^{-1}$ confirming the amide bond formation.

\section{Characterization of FA-conjugated TPT-loaded MSNs}

In this study, TPT-loaded MSNs were first prepared and then surface conjugation of FA on the surface of the NPs was performed. The FA present on the surface of NPs is expected to increase the targeting specificity toward the RB cells. The particle size of TMN was found to be $110 \pm 2.54 \mathrm{~nm}$ which was considerably increased to $168.5 \pm 1.65 \mathrm{~nm}$ after surface conjugation with FA. The increase in particle size was primarily attributed to the presence of FA on the surface of the NPs. The particle size of less than $200 \mathrm{~nm}$ would be beneficial to penetrate the tumor tissues which have irregular endothelium. The particles were perfectly spherical in nature and dispersed in the copper grid uniformly (Figure 2A). The particle size was consistent with the dynamic light scattering measurement.

\section{In vitro drug release}

In vitro drug release pattern helps in understanding the nature of drug-material interaction and its release kinetics in the simulated conditions. In general, release of encapsulated drug from the NPs depends on various factors such as nature of the materials, temperature, size, shape of the NP, and so on (Figure 2B). In this study, we have attempted to differentiate the release pattern of TMN and FTMN (impact of FA conjugation on the surface). It can be seen that TPT released in a relatively controlled manner from FTMN compared to that of TMN. The difference in the release of drug was primarily attributed to the presence of FA on the surface. The presence of FA on the surface might prevent the release of encapsulated drug from the core to the outer media. Approximately $30 \%$ of TPT released from TMN compared to $\sim 20 \%$ of drug released from FTMN after $24 \mathrm{~h}$ incubation. The same trend continued until the end of the study with $\sim 80 \%$ and $\sim 60 \%$ of drug release from TMN and FTMN, respectively, after $100 \mathrm{~h}$ incubation. The controlled release of drug will enhance the treatment modality in cancers.

\section{Cellular uptake efficiency of NPs in Y79 cells}

The targeting efficiency of TMN and FTMN in Y79 cells were investigated by cellular uptake studies. First, cellular uptake of NPs was studied by using flow cytometer. Rhodamine-B was loaded in the MSNs to track the uptake of NPs. As shown (Figure 3), FTMN showed a remarkably

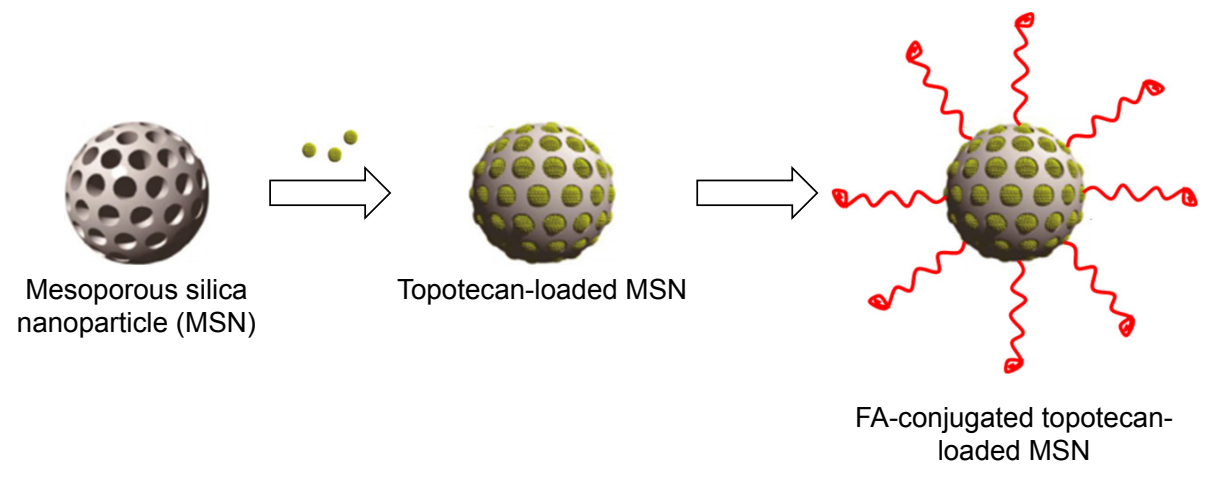

Figure I Schematic presentation of preparation of folic acid-conjugated mesoporous silica nanoparticles loaded with topotecan. Abbreviation: FA, folic acid. 
A

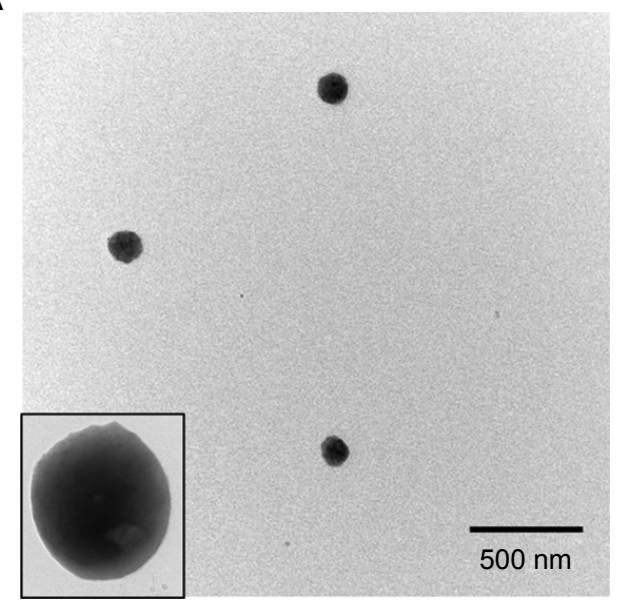

B

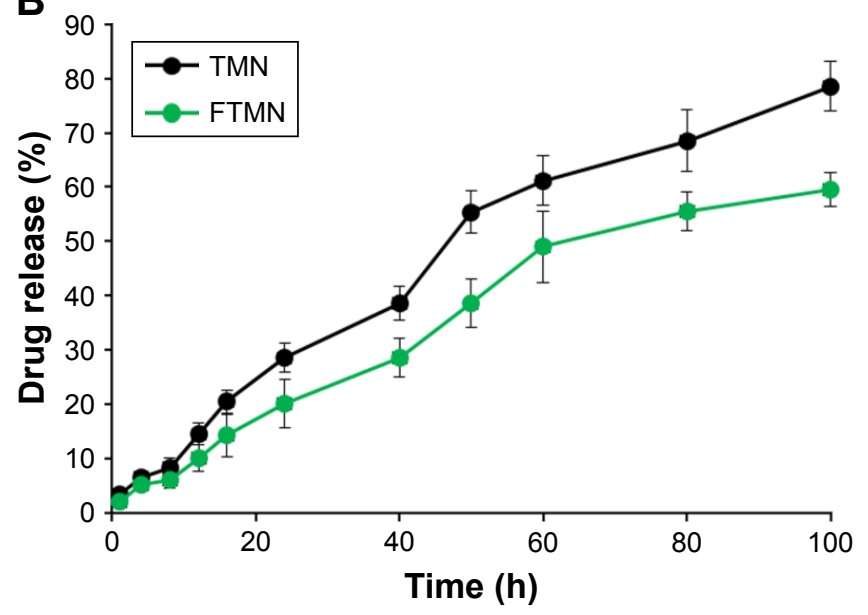

Figure 2 (A) TEM image of FTMN; (B) In vitro release profile of TPT from TMN and FTMN. The release study was performed in physiological media under $37^{\circ} \mathrm{C}$. Abbreviations: TEM, transmission electron microscopy; TPT, topotecan; TMN, TPT-loaded mesoporous silica nanoparticles; FTMN, TPT-loaded mesoporous silica nanoparticles surface conjugated with folic acid.

higher uptake in Y79 cancer cells compared to that of non-targeted TMN. The higher cellular internalization of FTMN was primarily attributed to the FA receptor-mediated endocytosis uptake mechanism..$^{22} \mathrm{TMN}$, however, might be internalized via a normal adsorption process.

The targeting efficiency of FTMN was further studied by CLSM. As seen (Figure 4), FTMN treated cell group showed a strong red fluorescence on the endolysosomal region compared to that of TMN. A strong red fluorescence on the cytoplasmic region is a proof that FTMN was internalized in a higher percentage than that of TMN. ${ }^{20}$ It has been

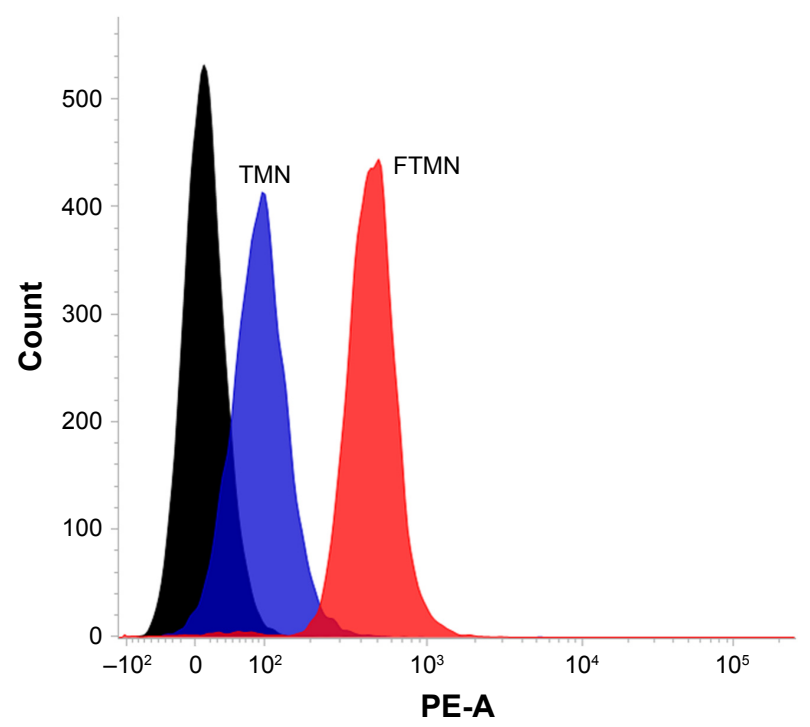

Figure 3 In vitro cellular uptake study of TMN and FTMN in Y79 cancer cells. The cellular uptake was studied by flow cytometer.

Abbreviations: PE-A, phycoerythrin; TPT, topotecan; TMN, TPT-loaded mesoporous silica nanoparticles; FTMN, TPT-loaded mesoporous silica nanoparticles surface conjugated with folic acid. reported that the internalized NPs in the lysosomes release the drug which will then travel to the desired pharmacological locations to exhibit its therapeutic effects. This finding is in agreement with the flow cytometer experiment. Overall, flow cytometer as well as CLSM clearly showed the superior targeting effect of FA-conjugated nanocarrier system.

\section{In vitro cytotoxicity assay}

The in vitro cytotoxicity assay of free drug and drug-loaded NPs was evaluated by MTT assay. First, cytotoxic effect of blank NP was studied in Y79 cancer cells. The results showed a high biocompatibility for the tested materials (Figure 5A). The cell viability was more than $>90 \%$ throughout all the concentrations $(200 \mu \mathrm{g} / \mathrm{mL})$. The excellent safety profile of blank nanocarrier is suitable for the systemic applications. Followed by this, cytotoxic potential of anticancer agents were studied in Y79 cancer cells. All the formulations showed a dose-dependent cytotoxic effect in the cancer cells. Especially, FTMN exhibited a remarkably higher anticancer effect in the cancer cells compared to that of any other formulations (Figure 5B). The $\mathrm{IC}_{50}$ value of FTMN was many fold smaller compared to that of either free drug or TMN indicating the excellent anticancer potential. The high cytotoxic effect of FTMN was primarily attributed to the enhanced cellular uptake of NPs in the cancer cells. It is well known that TPT is one of the components of therapeutic regimen in RB treatment; however, free form of TPT has side effects. Therefore, encapsulation of drug in the NPs will maintain the therapeutic potency of anticancer drugs while reducing its side effects. Our results clearly showed that nanoparticulate encapsulation of TPT exhibited superior 

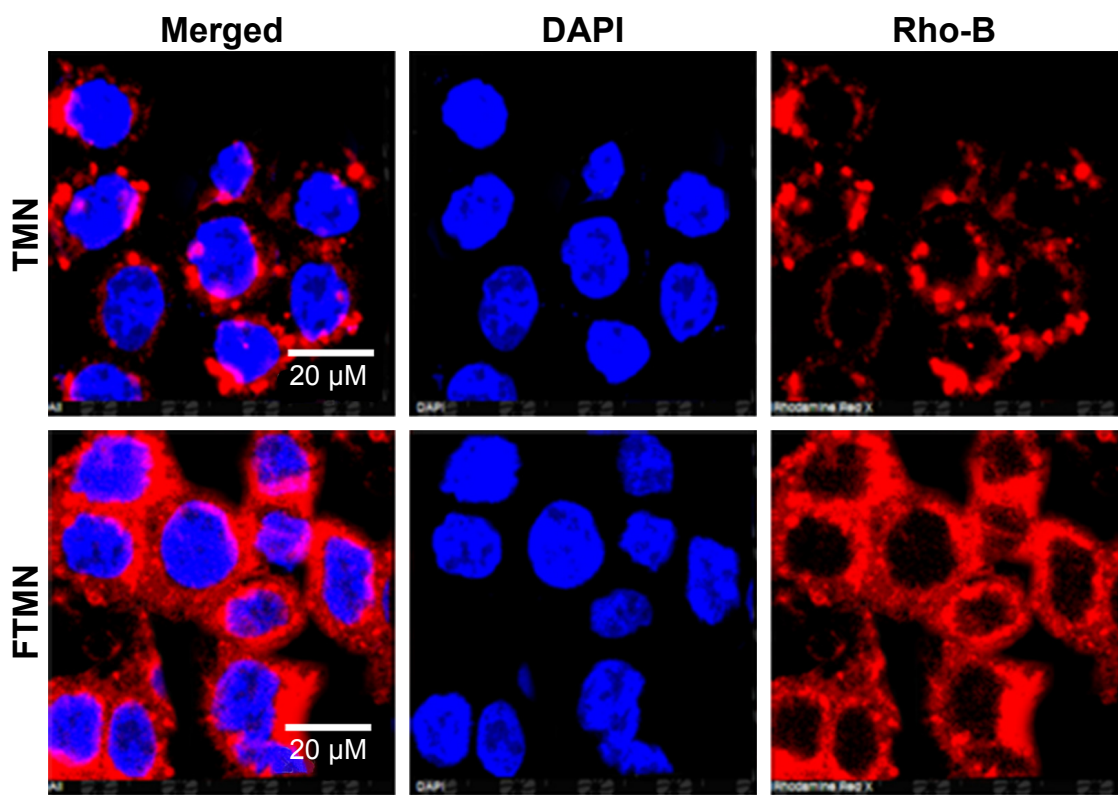

Figure 4 Colocalization of TMN and FTMN in Y79 cancer cells. The study was performed by CLSM. Scale bar: $20 \mu \mathrm{m}$.

Abbreviations: CLSM, confocal laser-scanning microscopy; DAPI, 4', 6-diamidino-2-phenylindole; TPT, topotecan; TMN, TPT-loaded mesoporous silica nanoparticles; FTMN, TPT-loaded mesoporous silica nanoparticles surface conjugated with folic acid; Rho-B, Rhodamine B.

anticancer efficacy in Y79 cancer cells compared to that of free drug or non-targeted NPs. ${ }^{23}$

We have performed an additional study on retinal ganglion cells (RGC-5; ATCC; Manassas, VA, USA) as a control cell line (Figure S2). As seen, free drug as well as drug-loaded formulations showed definitive cytotoxic effect in RGC- 5 cells although the intensity of cytotoxic action is lesser compared to that of the cancer cells. It must be noted that although formulations kill the RGC-5 cells at in vitro level, upon systemic administration, it is expected that most of the NPs will accumulate in the tumor cells via enhanced permeation and retention effect as well as FA receptor based-cancer targeting.

\section{Hoechst 33342 staining}

DNA fragmentation assay was performed to analyze the TPT-induced apoptosis by Hoechst 33342 staining (Figure 6). It can be seen that untreated cells maintained their typical morphology and present in large numbers. The TPT treatment, however, induced a typical apoptosis pattern. To be specific, FTMN showed a remarkably higher nuclear fragmentation confirming the higher apoptosis potential of FA-conjugated formulations than that of free drug or nontargeted NPs. Moreover, only fewer cells were left attached on the cover slip indicating the higher anticancer effect of the formulations than that of free drug or non-targeted NPs.
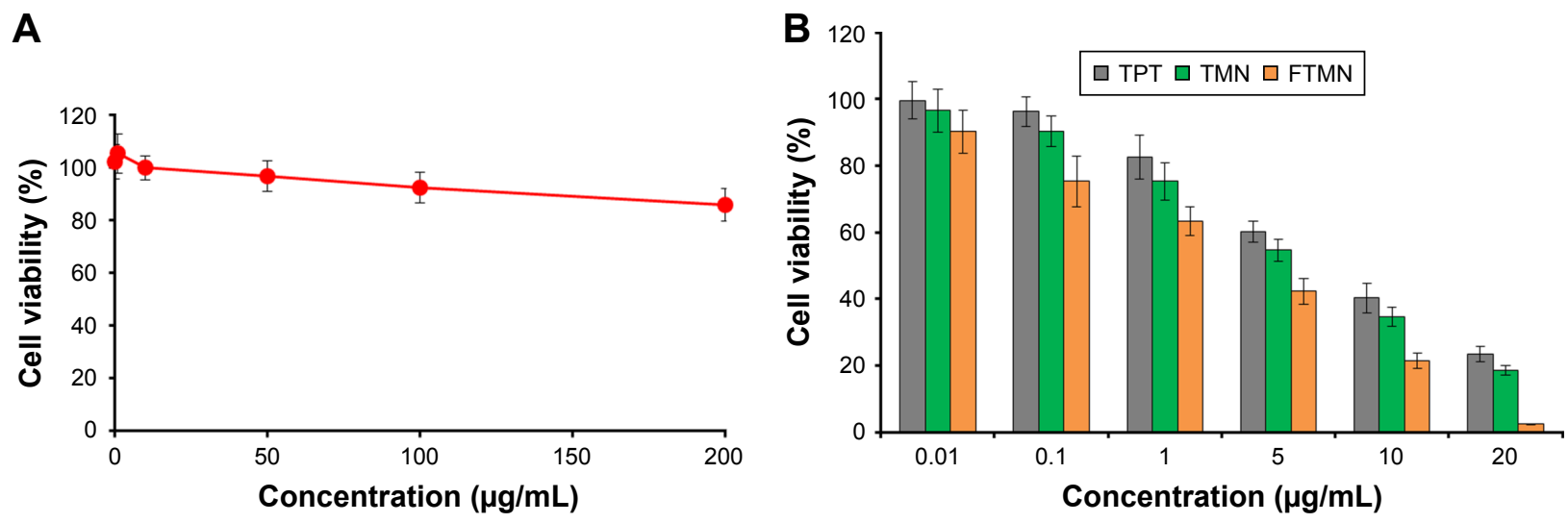

Figure 5 (A) Cytotoxicity analysis of blank nanoparticles; (B) In vitro cytotoxicity of blank NP, TPT, TMN, and FTMN in Y79 cancer cells after $24 \mathrm{~h}$ incubation. The cells were treated with respective formulations studied using MTT assay.

Abbreviations: NP, nanoparticle; TPT, topotecan; TMN, TPT-loaded mesoporous silica nanoparticles; FTMN, TPT-loaded mesoporous silica nanoparticles surface conjugated with folic acid. 


\section{Control}

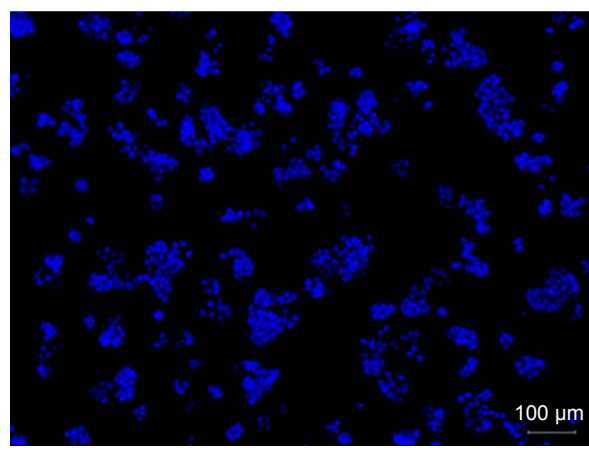

TMN

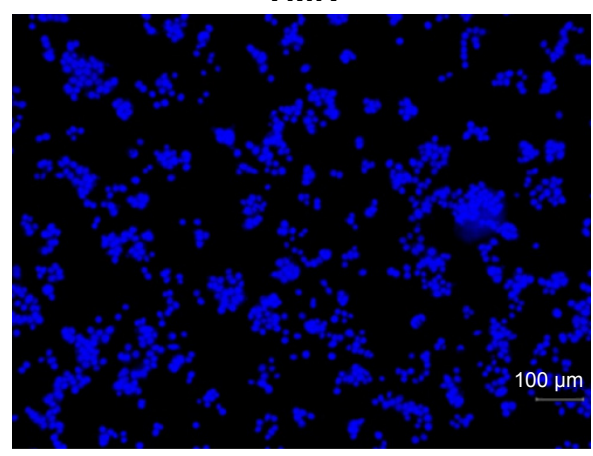

TPT

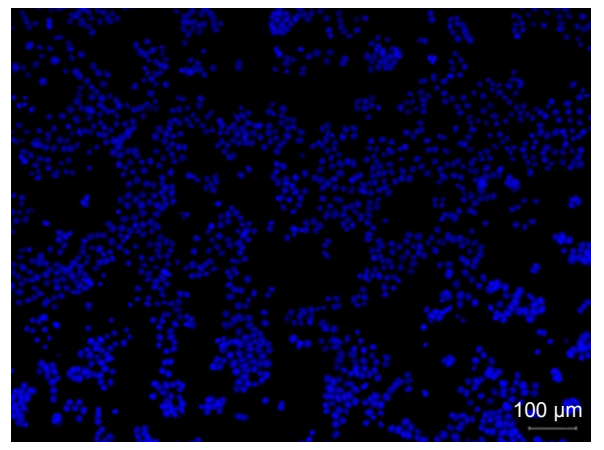

FTMN

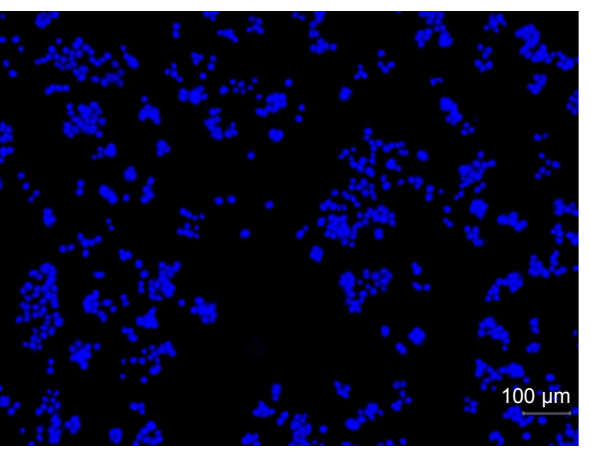

Figure 6 Hoechst 33342 based nuclear staining of Y79 cancer cells. The assay was performed by fluorescence microscopy.

The enhanced apoptosis effect of FTMN can be primarily attributed to the higher uptake potential of folic receptors overexpressed on the RB cells.

\section{Cellular morphology}

The cells were observed through optical microscope for the change in morphology after treatment with respective formulations (Figure 7A). The control cells maintained their typical shape, whereas formulation-treated cells showed definite changes in the morphology. Consistent with the cytotoxicity assay, FTMN-treated cells showed remarkably cancer cell death and the cells were largely rounded and fewer in number.

\section{Live/dead assay}

The cytotoxic potential of the synthesized TPT, TMN, and FTMN was further confirmed by live/dead assay (Figure 7B; Figure S3). The cells were treated with respective
A

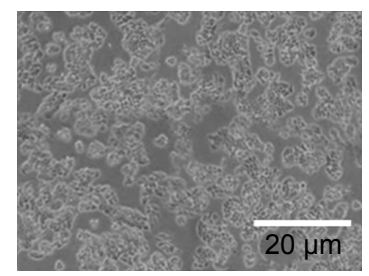

B

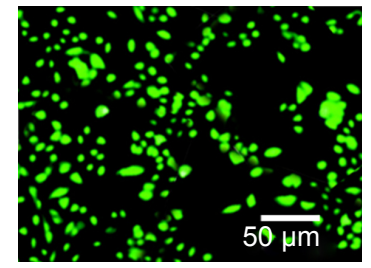

TPT
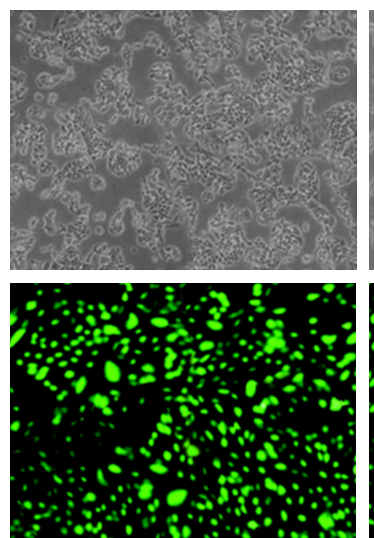

TMN
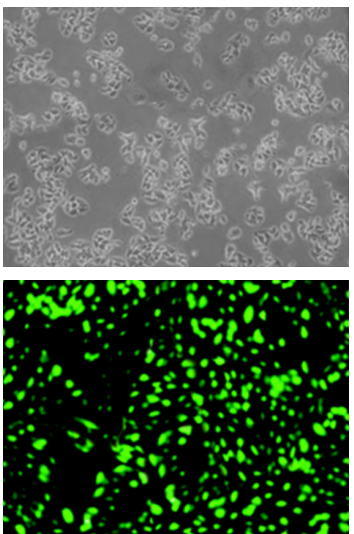

FTMN
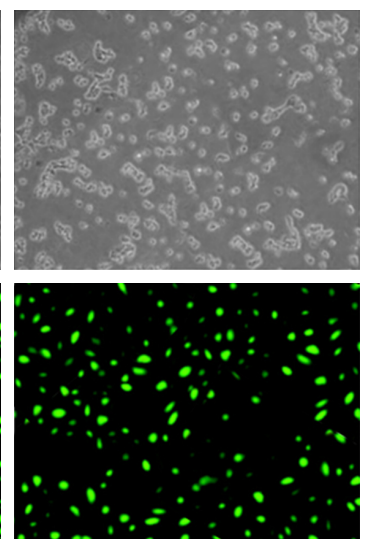

Figure 7 (A) Morphological imaging of Y79 cancer cells after treatment with TPT, TMN, and FTMN; (B) live dead assay of Y79 cancer cells after treating with respective formulations.

Abbreviations: TPT, topotecan; TMN, TPT-loaded mesoporous silica nanoparticles; FTMN, TPT-loaded mesoporous silica nanoparticles surface conjugated with folic acid. 
formulations and incubated for $24 \mathrm{~h}$. After drug incubation, cells were stained with calcein AM as a representative live cell marker. As seen, cells treated with free TPT showed reduced green fluorescence compared to that of untreated control. Consistent with the MTT assay, TMN and FTMN showed a remarkable decrease in the green fluorescence intensity indicating the superior anticancer effect of the optimized formulations. Again, the enhanced anticancer effect of FTMN might be due to the enhanced internalization of the FA in the NPs. It is generally known that calcein AM could enter the viable cells and gets converted into calcein in the presence of intracellular enzymes and thereby stains green color.
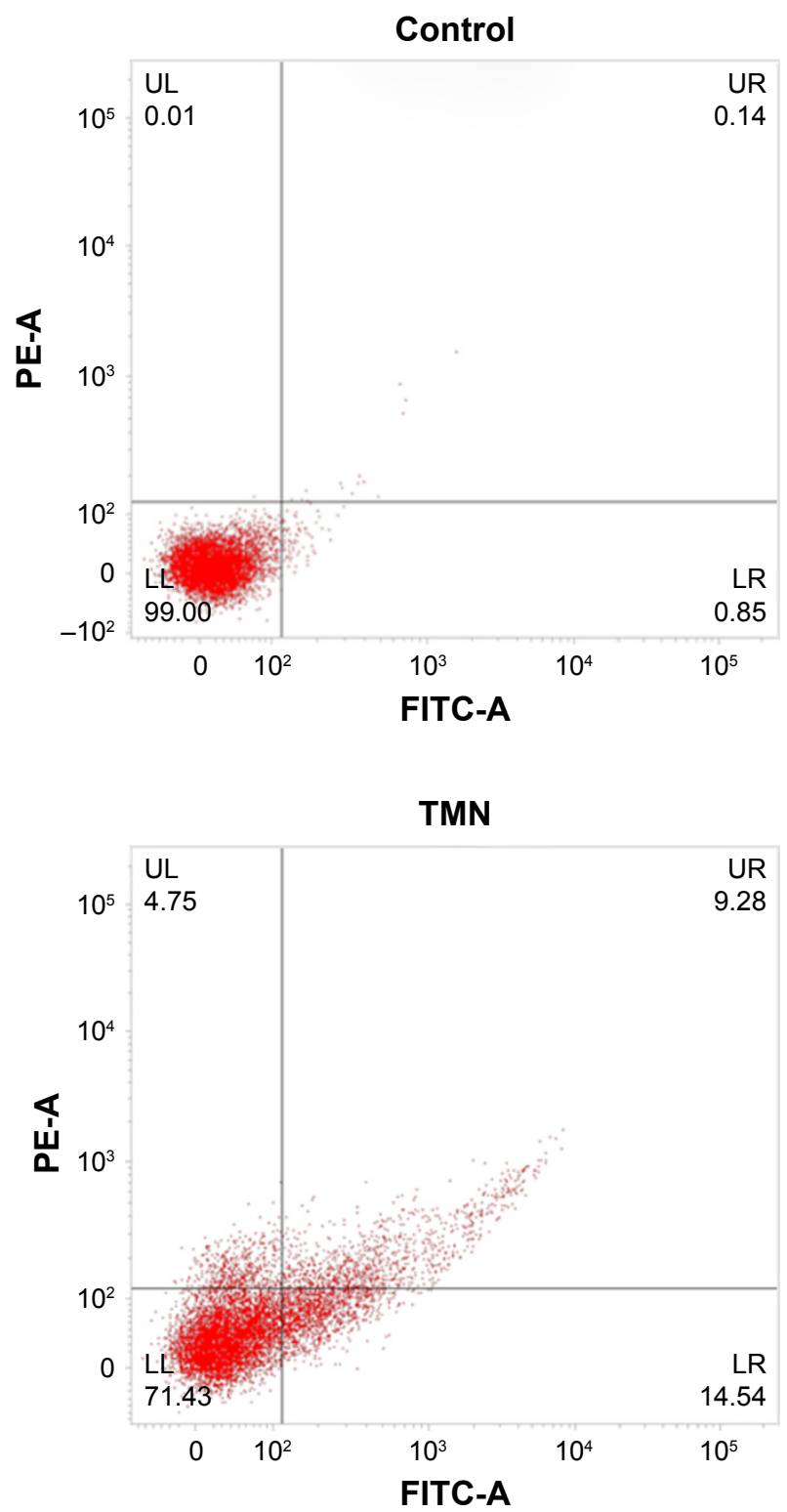

\section{Apoptosis assay}

The quantitative apoptosis assay was performed by Annexin- $V$ FITC/propidium iodide staining to investigate the apoptosis potential of free TPT, TMN, and FTMN, respectively (Figure 8). It is generally known that Annexin V detects the early apoptosis by phosphatidylserine externalization. The results clearly showed the apoptosis effect of individual formulations. No cells were present in early or late apoptosis in the untreated group, whereas $\sim 18 \%$ cells were present in the apoptosis quadrant after exposure with free TPT. The TMN increased the apoptosis cell percentage might due to better internalization compared to that of free drugs.
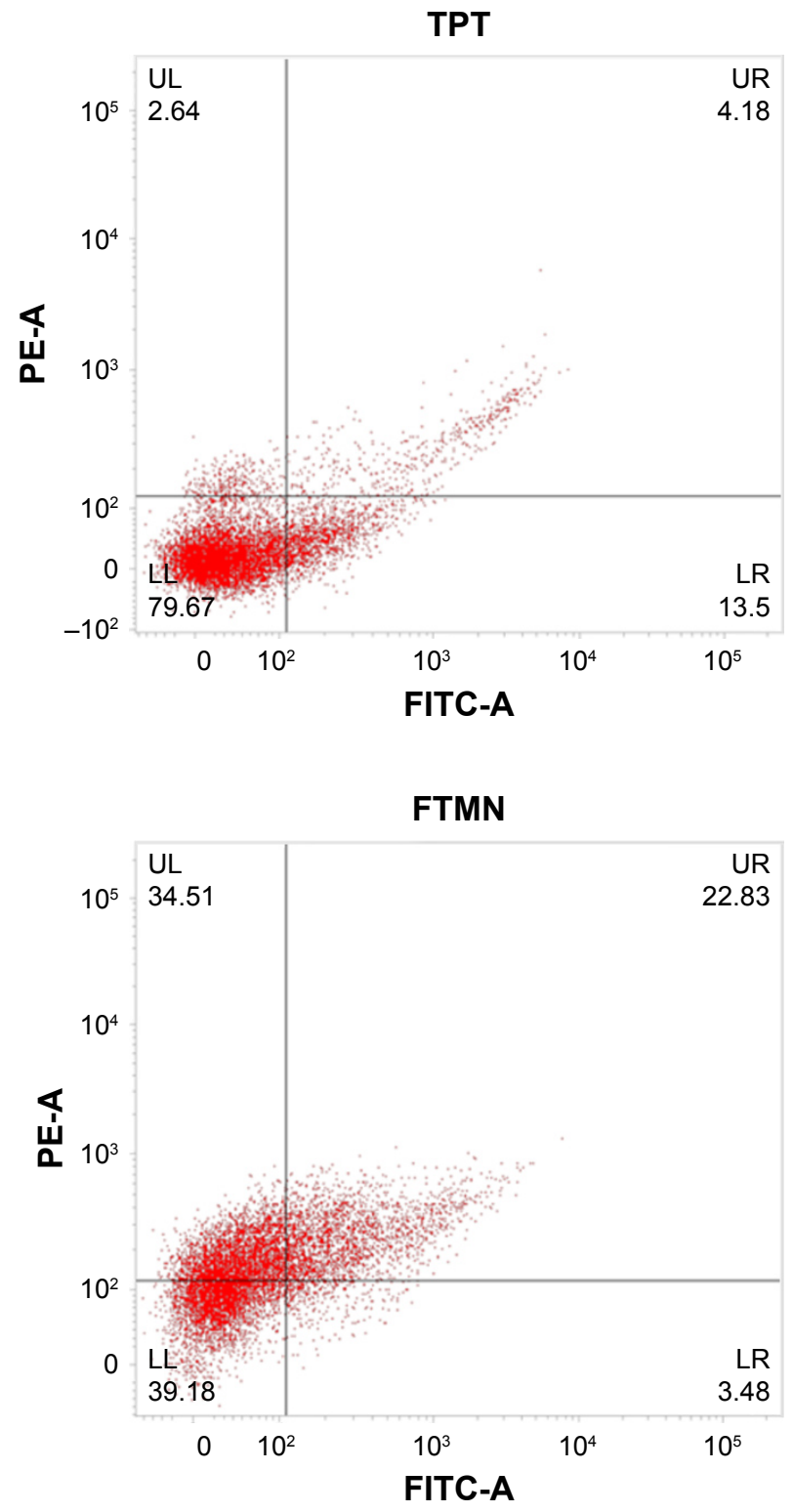

Figure 8 Induction of apoptosis in Y79 cancer cells by treatment with TPT, TMN, and FTMN. The cells were treated with respective formulations and stained with Annexin $V$ and $\mathrm{Pl}$.

Abbreviations: PE-A, phycoerythrin; TPT, topotecan; TMN, TPT-loaded mesoporous silica nanoparticles; FTMN, TPT-loaded mesoporous silica nanoparticles surface conjugated with folic acid; PI, propidium iodide; FITC, fluorescein isothiocyanate. 
Especially, FTMN showed the remarkable apoptosis of cancer cells with around $\sim 58 \%$ apoptosis. The results clearly indicate the superior anticancer effect of FTMN compared to that of any other formulations. The plausible reason for the superior apoptotic effect of FTMN was primarily attributed to the enhanced internalization of FA receptor-directed NPs. ${ }^{24}$ In addition, a sustained release pattern of drug to the cancer cells also induces the higher cancer cell death.

\section{In vivo antitumor efficacy}

The in vivo antitumor efficacy assay was performed in Y79-cancer cell bearing xenograft tumor model. The animal study was duly approved by the Institutional Animal Ethical Member Board, The 2nd Affiliated Hospital of Harbin Medical University, People's Republic of China. As seen (Figure 9A), tumor volume continued to grow in the untreated mice. In case of TPT, tumor volume was slightly less than that of control but not satisfactory. Results were improved in case of NP-based TMN due to the better accumulation of nanosized particles in the tumors. As expected, FTMN exhibited a remarkable reduction in the overall tumor volume compared to any other group. The excellent antitumor efficacy was primarily due to the targeting efficiency of FA toward the respective receptor in the tumor tissues that might increase the intracellular concentration of the chemotherapeutic drugs. The final tumor of FTMN was around $\sim 300 \mathrm{~mm}^{3}$ compared to that of $\sim 1,000 \mathrm{~mm}^{3}$ in case of control. The antitumor efficacy was further confirmed by the H\&E staining (Figure 9B). The mice injected with FTMN showed a few tumor cells compared to any other group. The control group retained their heteromorphism, including an irregular shape, darker stained nuclei, and nuclear atypia. By comparison with the groups treated with free drug, FTMN group showed dramatic decrease in tumor cell volume and nuclei. The results are concordant with the antitumor efficacy analysis.

\section{Conclusion}

In conclusion, FTMN were synthesized to enhance the therapeutic efficacy in RB cancers. The particles were nanosized and exhibited a sustained release of drug in the physiological conditions. The FA-conjugated nanoformulations exhibited a remarkable uptake in $\mathrm{RB}$ cells compared to that of non-targeted NPs. The results clearly indicated that receptor-mediated endocytosis is the mechanism of cellular internalization. The greater cellular uptake of FTMN resulted in significantly higher cytotoxic effect in Y79 cancer cells compared to that of other formulations. The results were well corroborated with the live/dead assay and nuclear fragmentation assay. Consistently, FTMN showed the remarkable apoptosis of cancer cells with around $\sim 58 \%$ apoptosis. Our results clearly showed that nanoparticulate encapsulation of TPT exhibited superior anticancer efficacy in Y79 cancer

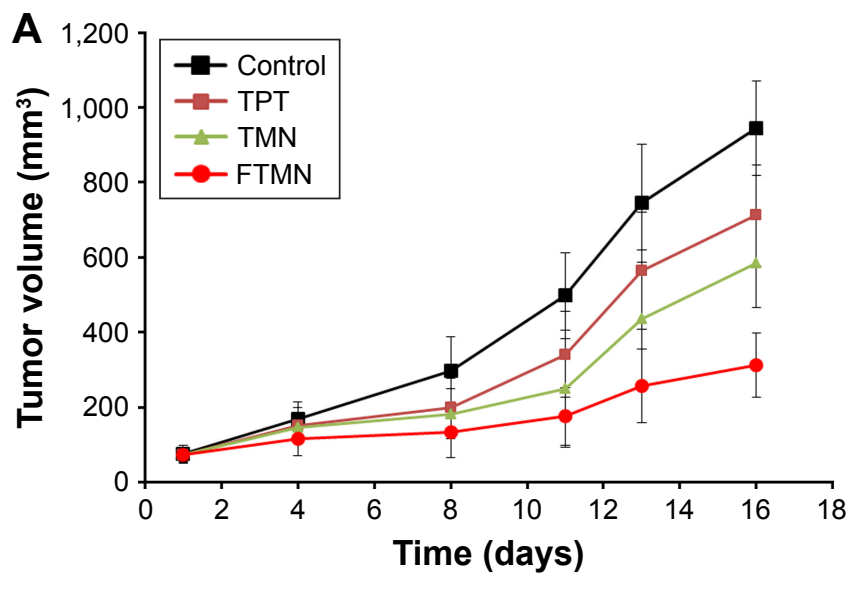

B

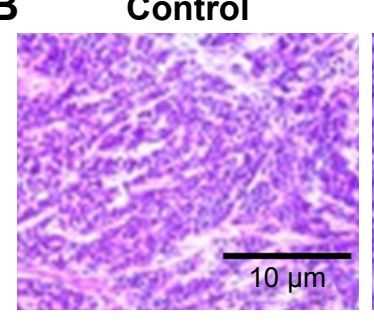

TPT

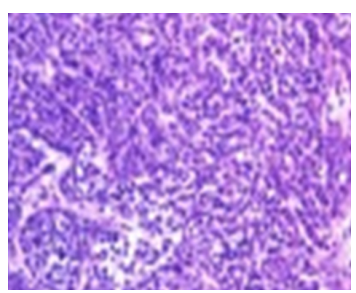

TMN

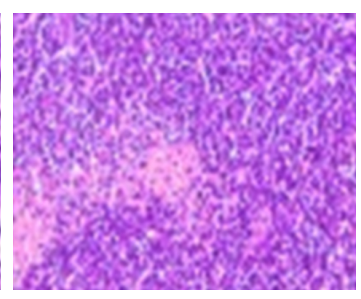

FTMN

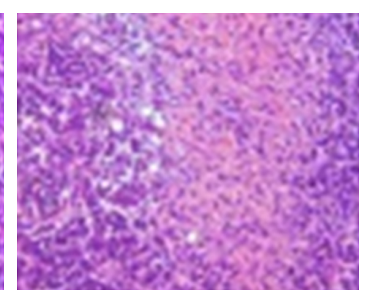

Figure 9 In vivo antitumor efficacy analysis in animal mode. (A) tumor volume and (B) H\&E staining.

Abbreviations: TPT, topotecan; TMN, TPT-loaded mesoporous silica nanoparticles; FTMN, TPT-loaded mesoporous silica nanoparticles surface conjugated with folic acid. 
cells compared to that of free drug or non-targeted NPs. As expected, FTMN exhibited a remarkable reduction in the overall tumor volume compared to any other group with less presence of tumor cells in histology staining. Overall, FA-conjugated nanoparticulate system could provide an effective platform for RB treatment.

\section{Acknowledgments}

This study was funded by the Natural Science Foundation of Heilongjiang Province of China (H201394) and the Doctoral Science Foundation of the 2nd Affiliated Hospital of Harbin Medical University.

\section{Disclosure}

The authors report no conflicts of interest in this work.

\section{References}

1. Abramson DH, Shields CL, Munier FL, Chantada GL. Treatment of retinoblastoma in 2015 agreement and disagreement. JAMA Ophthalmol. 2015;133(11):1341-1347.

2. Murphree AL, Villablanca JG, Deegan WF 3rd, et al. Chemotherapy plus local treatment in the management of intraocular retinoblastoma. Arch Ophthalmol. 1996;114(11):1348-1356.

3. Shields CL, Shields JA, Needle M, et al. Combined chemoreduction and adjuvant treatment for intraocular retinoblastoma. Ophthalmology. 1997;104(12):2101-2111.

4. Dunkel IJ, Lee TC, Shi W, et al. A phase II trial of carboplatin for intraocular retinoblastoma. Pediatr Blood Cancer. 2007;49(5):643-648.

5. Draper GJ, Sanders BM, Kingston JE. Second primary neoplasms in patients with retinoblastoma. Br J Cancer. 1986;53(5):661-671.

6. Abramson DH, Schefler AC. Update on retinoblastoma. Retina. 2004; 24(6):828-848.

7. Ramasamy T, Haidar ZS, Tran TH, et al. Layer-by-layer assembly of liposomal nanoparticles with PEGylated polyelectrolytes enhances systemic delivery of multiple anticancer drugs. Acta Biomater. 2014; 10(12):5116-5127.

8. Laurie NA, Gray NK, Zhang J, et al. Topotecan combination chemotherapy in two new rodent models of retinoblastoma. Clin Cancer Res. 2005;11(20):7569-7578.

9. Stewart CF, Zamboni WC, Crom WR, et al. Topoisomerase I interactive drugs in children with cancer. Invest New Drugs. 1996;14(1):37-47.
10. Zamboni WC, Stewart CF, Thompson J, et al. Relationship between topotecan systemic exposure and tumor response in human neuroblastoma xenografts. J Natl Cancer Inst. 1998;90(7):505-511.

11. Buitrago E, Höcht C, Chantada G, et al. Pharmacokinetic analysis of topotecan after intra-vitreal injection implications for retinoblastoma treatment. Exp Eye Res. 2010;91(1):9-14.

12. Lu J, Liong M, Zink JI, Tamanoi F. Mesoporous silica nanoparticles as a delivery system for hydrophobic anticancer drugs. Small. 2007;3(8):1341-1346.

13. Choi JY, Ramasamy T, Kim SY, et al. PEGylated lipid bilayer-supported mesoporous silica nanoparticle composite for synergistic co-delivery of axitinib and celastrol in multi-targeted cancer therapy. Acta Biomater. 2016;39:94-105.

14. Ramasamy T, Ruttala HB, Choi JY, et al. Engineering of a lipid-polymer nanoarchitectural platform for highly effective combination therapy of doxorubicin and irinotecan. Chem Commun. 2015;51:5758-5761.

15. Liong M, Lu J, Kovochich M, et al. Multifunctional inorganic nanoparticles for imaging, targeting, and drug delivery. ACS Nano. 2008; 2(5):889-896.

16. Lu J, Liong M, Li Z, Zink JI, Tamanoi F. Biocompatibility, biodistribution, and drug-delivery efficiency of mesoporous silica nanoparticles for cancer therapy in animals. Small. 2010;6(16):1794-1805.

17. Hom C, Lu J, Liong M, et al. Mesoporous silica nanoparticles facilitate delivery of siRNA to shutdown signaling pathways in mammalian cells. Small. 2010;6(11):1185-1190.

18. Crane LM, Arts HJ, van Oosten M, et al. The effect of chemotherapy on expression of folate receptor-alpha in ovarian cancer. Cell Oncol (Dordr). 2012;35(1):9-18.

19. Ramasamy T, Ruttala HB, Gupta B, et al. Smart chemistry-based nanosized drug delivery systems for systemic applications: a comprehensive review. J Control Release. 2017;258:226-253.

20. Leamon CP, Reddy JA. Folate-targeted chemotherapy. Adv Drug Deliv Rev. 2004;56(8):1127-1141.

21. Lu Y, Low PS. Immunotherapy of folate receptor-expressing tumors: review of recent advances and future prospects. J Control Release. 2003; 91(1-2):17-29.

22. Kansara V, Paturi D, Luo S, Gaudana R, Mitra AK. Folic acid transport via high affinity carrier-mediated system in human retinoblastoma cells. Int J Pharm. 2008;355(1-2):210-219.

23. Pan J, Feng SS. Targeted delivery of paclitaxel using folatedecorated poly(lactide)-vitamin E TPGS nanoparticles. Biomaterials. 2008;29(17):2663-2672.

24. Torchilin VP. Recent advances with liposomes as pharmaceutical carriers. Nat Rev Drug Discov. 2005;4(2):145-160. 


\section{Supplementary materials}

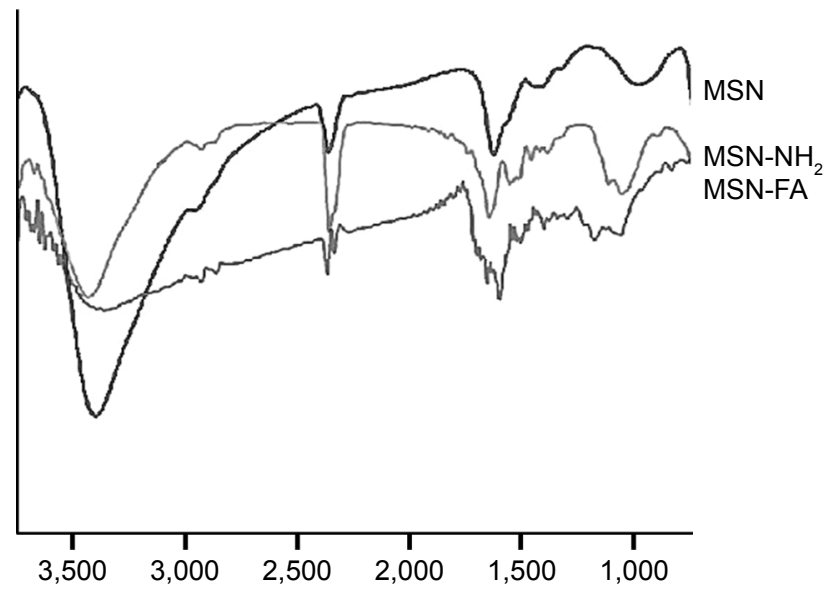

Figure SI FTIR spectra of MSN, MSN-NH $\mathrm{H}_{2}$ and MSN-FA.

Abbreviations: FTIR, Fourier transform infrared; MSNs, mesoporous silica nanoparticles; $\mathrm{MSN}-\mathrm{NH}_{2}$, mesoporous silica nanoparticles bonded with amide group; MSN-FA, mesoporous silica nanoparticles bonded with folic acid.

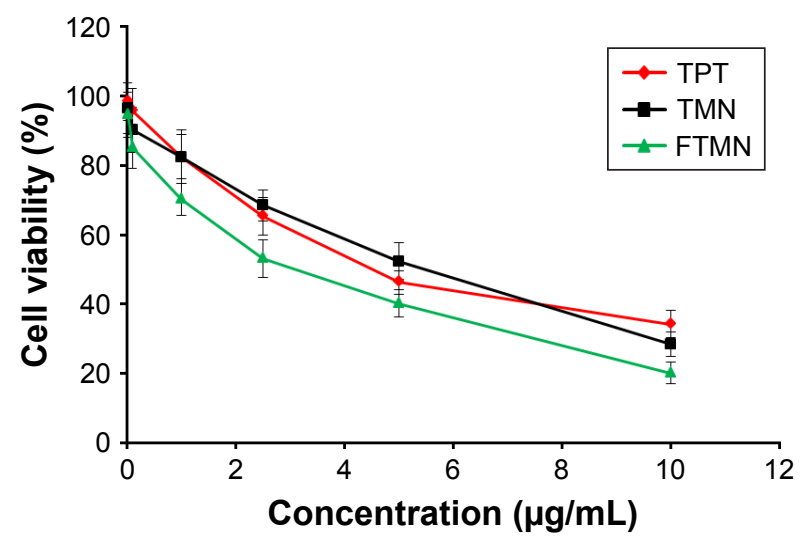

Figure $\mathbf{S 2}$ Cell viability of TPT, TMN, FTMN in RGC-5 cells.

Abbreviations: TPT, topotecan; TMN, TPT-loaded mesoporous silica nanoparticles; FTMN, TPT-loaded mesoporous silica nanoparticles surface conjugated with folic acid.

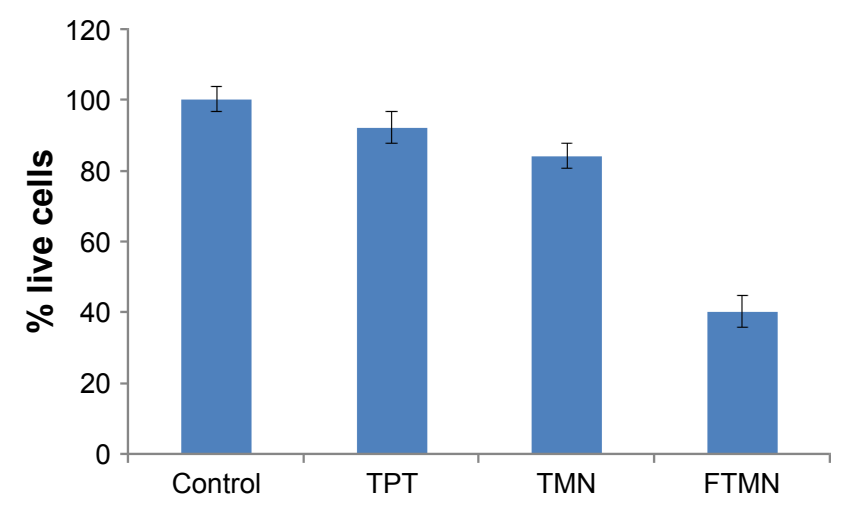

Figure S3 Live/dead assay of $\mathrm{Y} 79$ cells after treatment with respective formulations.
International Journal of Nanomedicine

\section{Publish your work in this journal}

The International Journal of Nanomedicine is an international, peerreviewed journal focusing on the application of nanotechnology in diagnostics, therapeutics, and drug delivery systems throughou the biomedical field. This journal is indexed on PubMed Central, MedLine, CAS, SciSearch $\AA$, Current Contents ${ }^{\circledR} /$ Clinical Medicine,

\section{Dovepress}

Journal Citation Reports/Science Edition, EMBase, Scopus and the Elsevier Bibliographic databases. The manuscript management system is completely online and includes a very quick and fair peer-review system, which is all easy to use. Visit http://www.dovepress.com/ testimonials.php to read real quotes from published authors. 